OPEN ACCESS

Edited by: Kette D. Valente, University of São Paulo, Brazil

Reviewed by:

Stéphane Auvin, Hôpital Robert Debré, France Edoardo Ferlazzo, Università degli studi Magna Græcia di Catanzaro, Italy

*Correspondence: Leilei Mao maoleilei@csu.edu.cn

Specialty section: This article was submitted to

Pediatric Neurology, a section of the journal Frontiers in Neurology

Received: 12 December 2018 Accepted: 08 April 2019

Published: 26 April 2019

Citation:

Peng P, Peng J, Yin F, Deng X, Chen $C$, He F, Wang $X$, Guang $S$ and

Mao L (2019) Ketogenic Diet as a Treatment for Super-Refractory Status Epilepticus in Febrile Infection-Related

Epilepsy Syndrome.

Front. Neurol. 10:423.

doi: 10.3389/fneur.2019.00423

\section{Ketogenic Diet as a Treatment for Super-Refractory Status Epilepticus in Febrile Infection-Related Epilepsy Syndrome}

\author{
Pan Peng ${ }^{1}$, Jing Peng ${ }^{1}$, Fei Yin ${ }^{1,2}$, Xiaolu Deng ${ }^{1}$, Chen Chen ${ }^{1}$, Fang $\mathrm{He}^{1}$, Xiaole Wang ${ }^{1}$, \\ Shiqi Guang ${ }^{1}$ and Leilei Mao ${ }^{1 *}$
}

${ }^{1}$ Department of Pediatrics, Xiangya Hospital of Central South University, Changsha, China, ${ }^{2}$ Hunan Intellectual and Developmental Disabilities Research Center, Xiangya Hospital of Central South University, Changsha, China

Background: Febrile infection-related epilepsy syndrome (FIRES) is a fatal epileptic encephalopathy associated with super-refractory status epilepticus (SRSE). Several treatment strategies have been proposed for this condition although the clinical outcomes are poor. Huge efforts from neurointensivists have been focused on identifying the characteristics of FIRES and treatment to reduce the mortality associated with this condition. However, the role of ketogenic diet (KD) in FIRES is not fully understood.

Methods: We performed a retrospective review of patients who met the diagnostic criteria of FIRES, SRSE, and were treated with KD between 2015 and 2018 at the Department of Pediatrics, Xiangya Hospital of Central South University. The following data were recorded: demographic features, clinical presentation, anticonvulsant treatment, timing and duration of KD and follow-up information. Electroencephalography recordings were reviewed and analyzed.

Results: Seven patients with FIRES were put on KD (5 via enteral route, and 2 via intravenous line) for SRSE in the PICU. The median age was 8. Four patients were male and 3 were female. Although patients underwent treatment with a median of 4 antiepileptic drugs and 2 anesthetic agents, the status epilepticus (SE) persisted for 7-31 days before KD initiation. After KD initiation, all patients achieved ketosis and SE disappeared within an average of 5 days (IQR 3.5), although there were minor side effects. In 6 patients, a unique pattern was identified in the EEG recording at the peak period. After initiation of $\mathrm{KD}$, the number of seizures reduced, the duration of seizure shortened, the background recovered and sleep architecture normalized in the EEG recordings. The early initiation of KD (at the onset of SE) in the acute phase of patients decreased the $\mathrm{mRS}$ score in the subsequent period $(p=0.012, r=0.866)$.

Conclusions: The characteristic EEG pattern in the acute phase promoted timely diagnosis of FIRES. Our data suggest that KD may be a safe and promising therapy for FIRES with SRSE, and that early initiation of KD produces a favorable prognosis. Therefore, KD should be applied earlier in the course of FIRES. Intravenous KD can be an effective alternative route of administration for patients who may not take KD enterally.

Keywords: ketogenic diet, febrile infection-related epilepsy syndrome, super-refractory status epilepticus, PICU, EEG 


\section{INTRODUCTION}

Febrile infection-related epilepsy syndrome (FIRES) is a rare epileptic encephalopathy of unknown etiology which occurs in patients without active epilepsy or underlying neurological disorders. It is characterized by new onset of refractory status epilepticus (SE) following a short nonspecific febrile illness (1). It is a biphasic disease, with an acute phase of refractory SE that always transforms into super-refractory SE (SRSE, i.e., SE that continues or recurs $24 \mathrm{~h}$ or more after the onset of anesthesia). This condition often requires intensive care and management, and it often progresses to a chronic phase which is characterized by refractory epilepsy without an intervening silent period. In the acute phase, the risk of mortality is high. Moreover, survivors are likely to live with a drug-resistant epilepsy and neuropsychological impairment. Nevertheless, few individuals do not experience neurologic sequelae or mild learning difficulties (2). Multiple therapeutic modalities have been reported for this disease, but most of them do not effectively control the high epileptic activity in such patients (3). Therefore, it is imperative to develop FIRES therapies with high efficacy.

Ketogenic diet (KD) contains high-fat, low-carbohydrate, and moderate protein content. It alters the primary cerebral energy supply from glucose to ketone bodies, mimicking the biochemical changes of starvation $(4,5)$. Although the exact mechanisms of KD's are not well-defined, it is thought that $\mathrm{KD}$ has antiepileptic activity, anti-inflammatory effect and neuroprotective activity (6-9) making it a therapeutic target for FIRES. In recent years, several small case studies have highlighted the value of diet in the treatment of FIRES. Results from such studies indicate that diet is not only suitable for acute patient management, but also for long-term management $(10,11)$. In April 2018, the International Ketogenic Diet Study Group have reached a consensus that KD is particularly useful for FIRES (12).

Despite fatal presentation, high disability and fatality rate in patients with FIRES, this syndrome is rarely diagnosed. Patient with FIRES are often diagnosed using the exclusion diagnosis method, and this explains why patients with FIRES are often diagnosed at a late stage due to lack of early disease markers. Currently, there is inadequate scientific data on the therapeutic efficacy of early initiation of KD for FIRES. Therefore, we aimed to explore strategies for timely recognition of FIRES through EEG recordings. Moreover, the KD therapy was given to 7 patients with FIRES and SRSE to investigate the efficacy and safety of $\mathrm{KD}$ as well as determine the effect of early $\mathrm{KD}$ treatment on the prognosis of FIRES patients.

\section{MATERIALS AND METHODS}

\section{Patients and Approvals}

We carried out a retrospective review of 7 patients diagnosed with FIRES $(13,14)$ and SRSE, and treated with KD at the Department of Pediatrics of Xiangya Hospital in Central South of China between 2015 and 2018. This study was approved by The Central South University Institutional Review Board.

\section{Clinical Data}

The following patient data was collected: demographics, general situations, history of seizures or SE, Glasgow Coma Scale (GCS), total hospital length of stay (LOS), ICU LOS, and antiepileptic drugs (AEDs) used before KD initiation and at discharge. Anesthetic agents, $\mathrm{SE}$ duration prior to $\mathrm{KD}, \mathrm{KD}$ ratio, type and duration of $\mathrm{KD}$, presence of ketones, and $\mathrm{KD}$ side effects were also obtained. KD therapy, seizure burden and modified Rankin Scale score (mRS) score at follow-up were collected in the outpatient neurology clinics. The clinical data were supplemented by a retrospective review of the electronic medical records (EMC).

Considering that SE has a subtle or no clinical manifestations in FIRES, its diagnosis requires EEG monitoring. Here, two approaches were adopted to define SE. Before hospital admission, SE was defined by continuous clinical or electrographic seizures lasting $5 \mathrm{~min}$ or more and/or recurrent episodes without recovery in between episodes (15). After admission, SE was defined by either a single seizure lasting $\geq 30 \mathrm{~min}$ or recurrent seizures totaling $\geq 30 \mathrm{~min}$ in any 1 -h period (16).

All EEG recordings were digital with time-locked synchronized video. Electrodes were positioned in accordance with the international 10-20 system of electrode placement. The EMG activity and ECG were also recorded. All patients were monitored with EEG for $2-15 \mathrm{~h}$ immediately after arrival at our PICU. The frequency of EEG monitoring varies, depending on available institutional resources and perceived clinical condition. All EEG recordings were analyzed individually by a boardcertified pediatric neurologist and two Asian Epilepsy Academy (ASEPA)-certified electroencephalographers. The following EEG features were analyzed: seizure burden, interictal and periodic epileptiform discharges, background activity, and sleep architecture. Five segments of EEG recordings were reviewed selectively to confirm the specific EEG pattern of FIRES and the effectiveness of $\mathrm{KD}$. We used the first recording at admission to our PICU as the 1\# epoch, the last recording prior to initiation of $\mathrm{KD}$ as the 2\# epoch. The recording at which SE disappeared after KD treatment was recorded as the $4 \#$ epoch, and the last recording before discharge as the $5 \#$ epoch. The $3 \#$ epoch was selected to divide the period between 2 and $4 \#$ approximately into two equal parts. All EEG data was stored in original format and was used for review.

Data were analyzed by reviewing of physician notes, laboratory findings, neuroimaging studies, EEG recordings and other supporting data.

\section{Diet Initiation}

Baseline laboratory examinations including complete blood counts, serum carnitine, chemistries, electrolytes, lipids, fatty acids were performed for all patients prior to KD initiation. These parameters were evaluated by a collaborative team of neurologists, critical care medicine personnel and nutrition experts to ensure there were no contraindications to $\mathrm{KD}$ therapy. KD was administered through two routes without a fasting period. All patients received enteral KD (EN KD) 
except 2 patients with severe gastrointestinal dysfunction who were placed on intravenous KD (IV KD). The commercially available ketogenic formula (Jiantong, Kinton medical food. Ltd, Guangzhou, China) with a fat to protein and carbohydrate (in gram scale) ratio of 4:1 were administered via enteral route and mixed with the current formula at the prescribed ketogenic ratio. IV KD comprised of commercially available fat emulsion with medium-chain triglycerides (Lipovenoes MCT, Sino-Swed Pharmaceutical Corp. Ltd, Wuxi, China), dextrose and amino acid solutions (Vamin, Sino-Swed Pharmaceutical Corp. Ltd, Wuxi, China).

The KD was initiated at a low ratio (about 2-3:1 in EN KD, 1:1 in IV KD) and then gradually advanced to a ratio of $3-4: 1$. The calorie intake of $\mathrm{KD}$ was restricted to $1 / 3$ of the estimated diet energy needs (75\% of recommended dietary allowance) initially and increased by $1 / 3$ every $1-3$ days (slower in IV KD) to full estimated diet energy. This step-by-step approach was adopted to prevent hypertriglyceridemia or hyperamylasemia induced by high intake or infusion of lipids. All dextrose was removed from fluids and all medications were changed to low-carbohydrate forms. Glucose-free solutions such as anticonvulsants and saline were prepared as required. Electrolytes, minerals, vitamins, and micronutrients were prepared at normal concentrations. The enteral preparation was given 6-8 times per day. IV KD was infused continuously over $20 \mathrm{~h}$ and stopped for $4 \mathrm{~h}$. When enteric-related complications such as gastrointestinal bleeding and enteroparalysis were resolved, $\mathrm{EN} \mathrm{KD}$ was initiated and the calorie intake was increased by $1 / 10-1 / 5$ of the estimated daily intake, adjusted after each 1-2 days to replace IV KD. Electrolytes, arterial blood gases, serum ketone bodies and glucose were measured once KD was initiated.

\section{Data Analysis}

Statistical analysis was performed using the Statistical Package for Social Science (IBM, SPSS Statistics Version 21). Proportions were calculated for continuous variables whereas medians and interquartile ranges (IQRs) were calculated for categorical variables. Pearson correlation analysis was applied for the duration of SE before KD initiation and $\mathrm{mRS}$ score at the latest follow-up (the normality test was completed).

\section{RESULTS}

\section{Clinical Characteristics}

In this series, all 7 patients ( 4 boys and 3 girls) were transferred from other health facilities and diagnosed with FIRES and SRSE in our PICU. All patients had a normal health and psychomotor development in their history. They did not have familial history of seizure or a known neurologic disease. The ages of patients at disease onset were between 1.5 and 13 years. The demographic data, clinical manifestations and management are described in Table 1. Out of 7 patients, 4 had a GCS score $<8$ at admission. The hospital LOS ranged from 35 to 86 days (median 46; IQR 18.5; mean $50.3 \pm 18.5$ ) and ICU LOS ranged from 19 to 61 days (median 32; IQR 15; mean $32.6 \pm 14.7$ ). Among them, no clear etiology could be determined despite extensive analyses ranging from microbiologic, metabolic and autoimmune causes, and early brain Magnetic Resonance Imaging (MRI).

All patients experienced a non-specific febrile illness prior to SE. Fever preceded the first seizures with a median duration of 4 days, ranging from 2 to 5 days. Shortly after onset of seizures, within $24 \mathrm{~h}$, the seizures rapidly progressed into SE or became frequent with loss of consciousness between the attacks. An obvious feature from the first EEG segment $(2-15 \mathrm{~h})$ was the heavy burden of seizure and most of them were either focal or subclinical with altered consciousness. Out of the 7 patients, 5 had seizures comprising $>50 \%$ in a 1 -h period. Notable EEG parameters are described below and summarized in Table 2.

We identified a specific ictal pattern of the seizures in 6 patients. This seizure pattern consisted of sharp (or spike) wave and/or sharp (or spike) slow wave complex of low to moderate amplitude, arising from unilateral or bilateral focal area, spreading to the same hemisphere and/or bilaterally with higher amplitude and faster/slower frequency, sometimes shifting from one hemisphere to the contralateral (Figure 1). Interictally, some patients displayed unidentified sleep architecture and diffuse delta-theta background slowing, with or without multifocal sporadic or periodic epileptiform discharges (sharp or spike waves, complex waves). These features might be undistinguishable from non-intermittent ictal activity in severe cases.

All patients experienced SE for 7-31 days (median 12; IQR 11 ; mean $16.6 \pm 9.5$ ) before $\mathrm{KD}$ initiation. In spite of the conventional management with a median of four AEDs and two continuous anesthetic agents before initiation of $\mathrm{KD}$, the nearly continuous electrical or electroclinical seizures were poorly controlled or reappeared when anesthetic agents were tapered. Moreover, 2 patients received several anesthetics assistants (Fentanyl or its derivatives, Cisatracurium Besilate, Vecuronium) to control volcanic seizures. Three patients were under full mechanical ventilation due to severe respiratory depression. Similarly, other therapies such as intravenous immunoglobulin (IVIg) (in all patients) and Methylprednisolone (in 5 patients) were ineffective before initiation of KD. Before initiation of KD, most of the patients (except three) has stopped immunotherapy before or early after admission at our hospital. The three patients were continued on a low-dose steroid therapy even after $\mathrm{KD}$ initiated.

\section{Outcomes After KD Treatment}

All patients achieved resolution of SRSE (Table 3) within a median of 5 days (IQR 3.5) after initiation of KD. The time to ketosis (defined as the level of serum $\beta$-hydroxybutyrate $>1 \mathrm{mmol} / \mathrm{L}$ ) of 6 of 7 patients ranged from 1 to 6 days, but 1 of 7 patients required 11 days to achieve ketosis after $\mathrm{KD}$ initiation. According to the EEG data, the seizure burden reduced, duration of seizure shortened, background recovered, and sleep architecture normalized gradually after KD initiation. Besides, 4 of 7 patients were weaned off general anesthetics within 15 days of KD initiation whereas one of them stopped anesthesia infusion before KD initiation to reduce respiratory secretions. 
TABLE 1 | Patient characteristics and treatment.

\begin{tabular}{|c|c|c|c|c|c|c|c|}
\hline & Patient 1 & Patient 2 & Patient 3 & Patient 4 & Patient 5 & Patient 6 & Patient 7 \\
\hline Antecedent febrile infection & FUO & URTI & URTI & GE & URTI & FUO & FUO \\
\hline Latent period* & 3 & 5 & 4 & 4 & 2 & 5 & 3 \\
\hline Admit GCS score & 4 & 3 & 9 & E4VTM1 & E1VTM1 & Confusion & Confusion \\
\hline Duration of SE before KD, $d$ & 31 & 12 & 11 & 15 & 11 & 29 & 7 \\
\hline $\begin{array}{l}\text { No. of AEDs received } \\
\text { before KD }\end{array}$ & 5 & 4 & 4 & 5 & 3 & 4 & 4 \\
\hline AEDs received before $\mathrm{KD}$ & $\begin{array}{l}\text { PHB, CZP, VPA, } \\
\text { TPM, OXC }\end{array}$ & $\begin{array}{l}\text { VPA, LEV, OXC, } \\
\text { NZP }\end{array}$ & $\begin{array}{l}\text { PHB, VPA, TPM, } \\
\text { LEV }\end{array}$ & $\begin{array}{l}\text { PHB, CZP, VPA, } \\
\text { TPM, OXC }\end{array}$ & VPA, TPM, LEV & $\begin{array}{l}\text { VPA, LEV, TPM, } \\
\text { OXC }\end{array}$ & $\begin{array}{l}\text { VPA, LEV, TPM, } \\
\text { OXC }\end{array}$ \\
\hline $\begin{array}{l}\text { No. of anesthetic agents } \\
\text { before KD }\end{array}$ & 2 & 2 & 2 & 2 & 2 & 1 & 1 \\
\hline Anesthetic agents before KD & Midazolam, Propofol & $\begin{array}{l}\text { Midazolam, } \\
\text { Propofol }\end{array}$ & $\begin{array}{l}\text { Midazolam, } \\
\text { Propofol }\end{array}$ & $\begin{array}{l}\text { Midazolam, } \\
\text { Propofol }\end{array}$ & $\begin{array}{l}\text { Midazolam, } \\
\text { Propofol }\end{array}$ & Midazolam & Midazolam \\
\hline $\begin{array}{l}\text { Anesthetics assistants } \\
\text { before KD }\end{array}$ & $\begin{array}{l}\text { Suf, Cisatracurium } \\
\text { Besilate, Vecuronium }\end{array}$ & / & / & / & Fentanyl & / & / \\
\hline Other treatments before KD & MP, IVIG, PE & MP, IVIG & IVIG & MP, IVIG & MP, IVIG, PE & IVIG & MP, IVIG \\
\hline AMV & Yes & No & No & Yes & Yes & No & No \\
\hline KD ratio & $1.2: 1 \rightarrow 4: 1$ & $1.5: 1 \rightarrow 3.2: 1$ & $2: 1 \rightarrow 2.5: 1$ & $1.5: 1 \rightarrow 3: 1$ & $0.7: 1 \rightarrow 4: 1$ & $2: 1 \rightarrow 3: 1$ & $3: 1 \rightarrow 4: 1$ \\
\hline Type of KD & Parental & Enteral & Enteral & Enteral & Parental & Enteral & Enteral \\
\hline
\end{tabular}

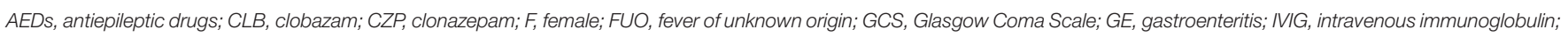

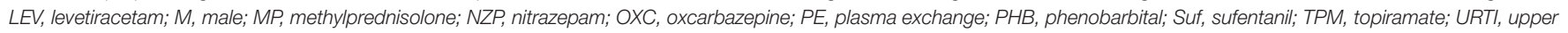
respiratory tract infection; VPA, valproic acid.

* Latent period: the days between the febrile illness and onset of seizures.

The two patients who received intravenous $\mathrm{KD}$ were changed to enteral diet route gradually after resolution of SRSE.

There was no severe acidosis $\left(\mathrm{HCO}_{3}^{-}<17 \mathrm{mmol} / \mathrm{L}\right)$ or hypoglycemia during KD therapy. Seven patients showed mild anomalies in their laboratory tests (Table 3). Of the 7 patients, 3 developed hyperlipemia or experienced diarrhea while 1 of 7 patients developed both conditions. Serum amylase increased to $389.7 \mathrm{mmol} / \mathrm{L}$ in one patient who was given IV $\mathrm{KD}$, but normalized after adjustment of the proportion of KD ingredients, carbohydrate and by changing to enteral route. On discharge, all patients were followed up at the pediatric neurology outpatient clinics (Table 3). The number of AEDs used at hospital discharge ranged from 3 to 4 drugs. Duration from the time of discharge to the most recent follow-up ranged from 6 to 40 months. No patient remained on $\mathrm{KD}$ at the latest follow-up. Of 7 patients, one patient continued the diet for nearly 1 year compared to 6 patients who discontinued KD in 3 months. Among them, one patient had a recurrence of intractable epilepsy after about 2 weeks of treatment. The patient was, therefore, put on therapy of vagus nerve stimulation (VNS). The remaining patients were free of seizure attacks during the acute phase ( 4 were seizurefree, and one had intermittent seizures). At the time when KD was stopped, 4 of 7 patients had a mRS score $\leq 3$, and the rate increased to 6 of 7 at the latest follow-up. A shorter duration of SE before KD initiation reflected a lower mRS score in the subsequent period ( $p=0.012, r=0.866)$. The recovery of phycomotor function was diverse among the patients, 3 cases (patient 2,3,7) were almost cured, 1 case (patient 5) had a significant improvement, and 3 cases (patient 1,4,6) showed a moderate improvement.

\section{DISCUSSION}

Although the clinical manifestations and disease course of FIRES have been well-defined in the literatures, this sudden and severe epileptic encephalopathy is still challenging. Patient with FIRES are often diagnosed using the exclusion diagnosis method, and this explains why patients with FIRES are often diagnosed at a late stage due to lack of early disease markers. FIRES is characterized by repeated and prolonged seizures, and patients with FIRES are always in prolonged pharmacologic coma which is often accompanied with medical complications. It is important to develop available diagnostic tools to facilitate prompt diagnosis and design effective therapies to improve the management and prognosis of this condition.

This study found that EEG monitoring is an effective method of diagnosing FIRES based on recordings of explosive seizures (most were subclinical) with high frequency. At the peak hours of SE, we identified a characteristic EEG pattern consisting of focal ictal activity of small to moderate amplitude at the onset of the seizure, which evolved into higher amplitude and faster/slower frequency, spreading unilaterally and/or bilaterally, sometimes shifting from one hemisphere to the contralateral. These features can be used for early recognition and intervention of FIRES.

Currently, there are no effective treatments for FIRES. Several case studies have shown that AEDs and immunological therapy do not satisfactorily control FIRES. And other studies have raised that the prolonged burst-suppression coma may be associated with a more severe course (2). Interestingly, some children with FIRES displayed good response to KD. Nine studies including 26 patients have reported this phenomenon $(7,10,13,17-22)$. The 


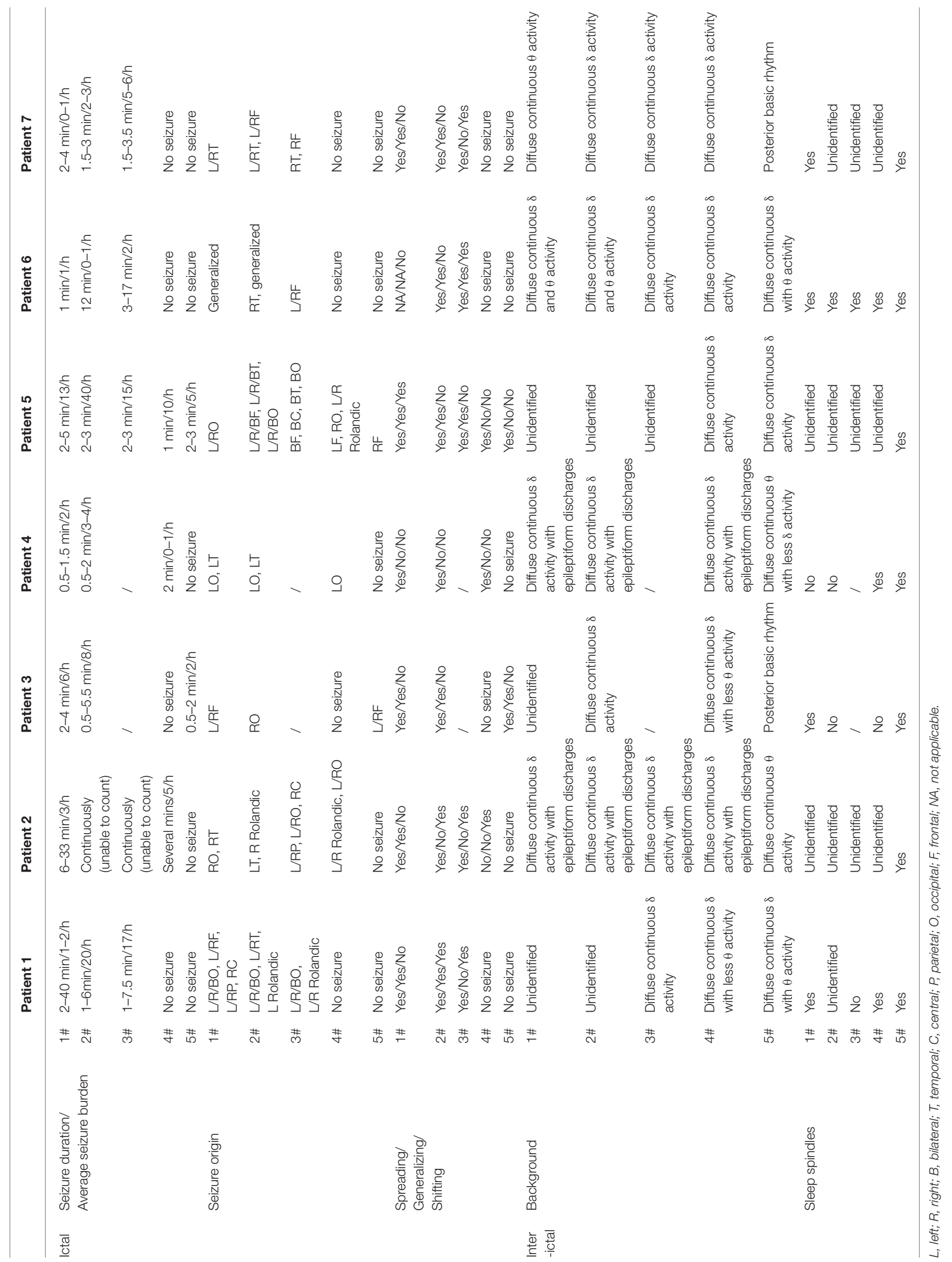




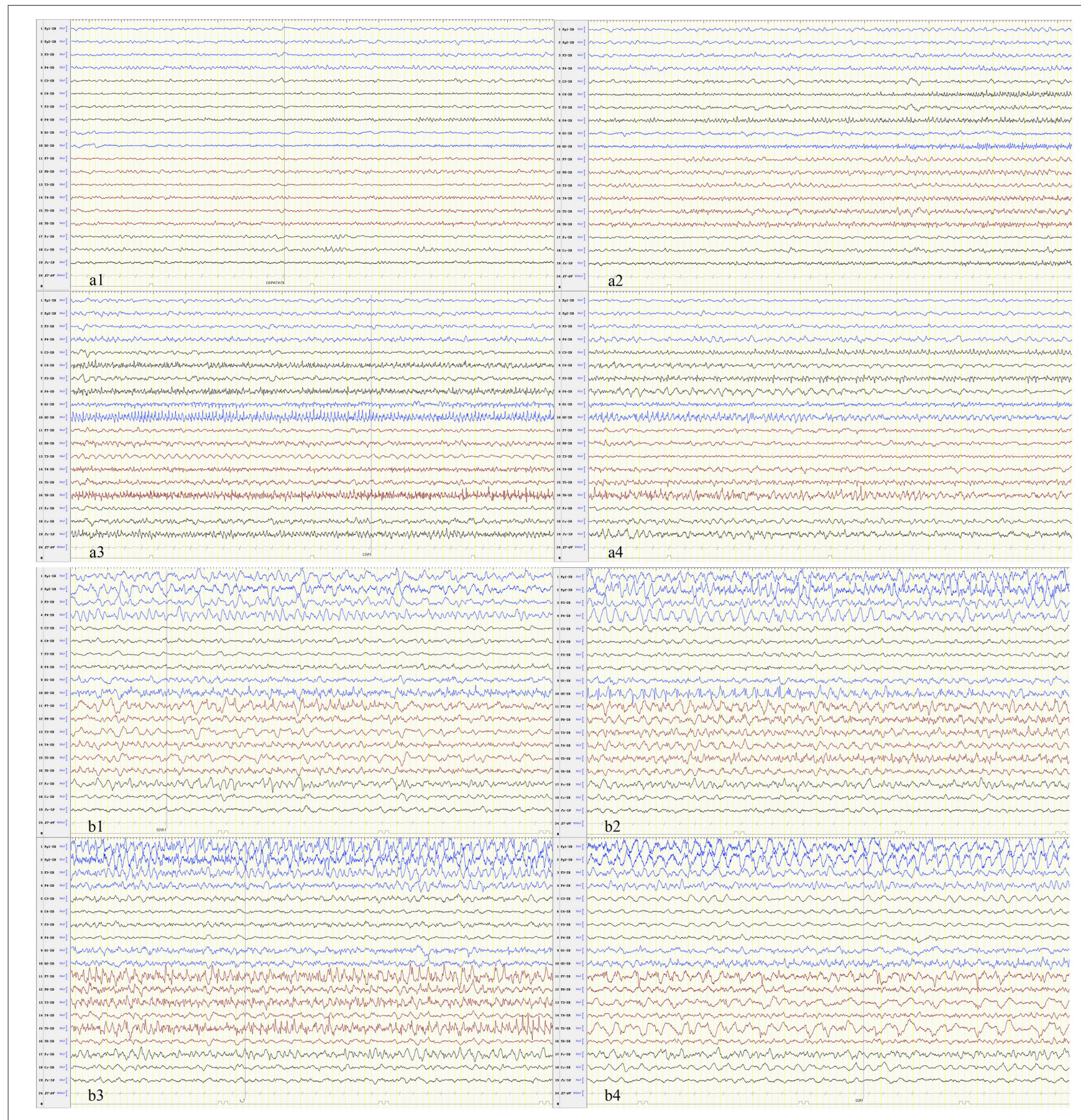

FIGURE 1 | The seizure begins in the focal or multifocal regions with spread to the unilateral or bilateral hemisphere and shifting from one hemisphere to the contralateral. (a) Patient 1 and (b) patient 6.

effective rate ( $>50 \%$ seizure reduction) was $62 \%$. In this study of 7 children with FIRES and SRSE, we found that immunotherapy and AEDs could not control this condition, but KD treatment produced significant therapeutic efficacy and safety, which is consistent with previous reports.

It is worth noting that the clinical presentation of patients in this study shows that early initiation of $\mathrm{KD}$ improves outcomes of FIRES. In all patients, the frequency of seizures at the last follow-up was similar, and seizures were reduced by $\geq 75 \%$ in all patients except one who converted to VNS. However, their functional outcomes were dissimilar. A shorter duration of SE in the acute phase of patients indicated a lower mRS score in the subsequent period $(p=0.012, r=0.866)$. Patients $(2,3,5,7)$ who had an SE duration of $<15$ days before initiation of $\mathrm{KD}$ had a 
TABLE 3 | Patient in-hospital and long-term outcomes.

\begin{tabular}{|c|c|c|c|c|c|c|c|c|}
\hline & & Patient 1 & Patient 2 & Patient 3 & Patient 4 & Patient 5 & Patient 6 & Patient 7 \\
\hline \multicolumn{9}{|c|}{ Clinical outcomes } \\
\hline \multicolumn{2}{|c|}{ Time to ketosis, $d$} & 3 & 2 & 11 & 1 & 5 & 1 & 3 \\
\hline \multicolumn{2}{|c|}{$\begin{array}{l}\text { Length of anesthesia after KD } \\
\text { initiation, } d\end{array}$} & 23 & 29 & 14 & 11 & 24 & 7 & NA \\
\hline \multicolumn{2}{|l|}{ Adverse events } & $\begin{array}{l}\text { Hyperlipidemia, } \\
\text { transient } \\
\text { hyperamylasemia }\end{array}$ & $\begin{array}{l}\text { Hyperlipidemia, } \\
\text { diarrhea }\end{array}$ & Diarrhea & Hyperlipidemia & Hyperlipidemia & Diarrhea & Diarrhea \\
\hline \multicolumn{2}{|l|}{ Total LOS, $d$} & 86 & 48 & 36 & 38 & 63 & 46 & 35 \\
\hline \multicolumn{2}{|l|}{ ICU LOS, $d$} & 61 & 32 & 19 & 23 & 40 & 33 & 20 \\
\hline \multirow[t]{2}{*}{ Seizure burden } & $\begin{array}{l}\text { At the end of } \\
\mathrm{KD}\end{array}$ & $\begin{array}{l}\text { Monthly seizures } \\
\text { (after controlled for } \\
1 \text { month) }\end{array}$ & No seizure & No seizure & $\begin{array}{l}\text { Daily seizures } \\
\text { (after controlled } \\
\text { for } 2 \text { weeks) }\end{array}$ & $\begin{array}{l}\text { Monthly seizures } \\
\text { (after controlled } \\
\text { for } 2 \text { months) }\end{array}$ & No seizure & No seizure \\
\hline & $\begin{array}{l}\text { At most } \\
\text { recent } \\
\text { follow-up }\end{array}$ & $\begin{array}{l}\text { Monthly } \\
\text { seizures }\end{array}$ & No seizure & $\begin{array}{l}\text { Monthly } \\
\text { seizures }\end{array}$ & $\begin{array}{l}\text { Monthly } \\
\text { seizures }\end{array}$ & $\begin{array}{l}\text { Monthly } \\
\text { seizures }\end{array}$ & $\begin{array}{l}\text { Monthly } \\
\text { seizures }\end{array}$ & No seizure \\
\hline \multirow[t]{2}{*}{ mRS score } & $\begin{array}{l}\text { At the end of } \\
\mathrm{KD}\end{array}$ & 4 & 0 & 0 & 4 & 3 & 4 & 0 \\
\hline & $\begin{array}{l}\text { At most } \\
\text { recent } \\
\text { follow-up }\end{array}$ & 4 & 0 & 0 & 3 & 1 & 3 & 0 \\
\hline
\end{tabular}

mRS, modified Rankin Scale; NA, not applicable (because the anesthesia had been withdrawn before KD initiation in patient 7).

better prognosis with mRS scores $\leq 3$ at the end of $\mathrm{KD}$ and $\leq 1$ at the last follow up. In these patients, it is important to recognize FIRES symptoms early using specific EEG pattern and initiate KD treatment. KD therapy resolved SRSE successfully unlike other treatments (immunotherapy, four or more AEDs, and at least one general anesthetic agent). This indicates that $\mathrm{KD}$ can prevent damage from excitotoxicity processes, inhibit secondary processes induced by initial excitotoxicity, prevent multiple complications due to prolonged anesthesia and unconsciousness, increase alertness of patients and decrease the need for invasive respiratory support.

Generally, KD can be administered through enteral route (by mouth or tubes). But patients with conditions such as FIRES often suffer from gastrointestinal problems caused by prolonged use of AEDs, general anesthetics and mechanical ventilation. Some of the gastrointestinal problems include vomiting, severe diarrhea, and intestinal bleeding. Therefore, administration of drugs using enteral route may worsen these complications. In fact, in the presence of these conditions, enteral intake of $\mathrm{KD}$ is compromised and ketosis is impaired, making $\mathrm{KD}$ diet unsafe and inefficient (23). In this study, KD was given as parenteral nutrition to 2 children with FIRES and SRSE (patient 1 and 5) who required bowel rest due to upper gastrointestinal hemorrhage or gastroplegia. Thereafter, they were converted to enteral diet successfully. This study has demonstrated that KD can be effectively applied to patients with intestinal failure who need to start KD early.

The major limitations of this study should be discussed. This is a retrospective study containing a small number of patients. Considering the severity of patients with SRSE, we were unable to include a group of patients with SRSE and FIRES who were not put on KD treatment during the same period for comparison. Moreover, a combination of treatments comprising AEDs, immunotherapy and $\mathrm{KD}$ was used, making it difficult to isolate the exact benefit of a single intervention while excluding other treatments. Nevertheless, considering the rapid response (resolution of SE in clinical course and improvement of EEG features on assistant examination) in acute phase and good prognosis of patients in this study, we conclude that KD treatment is effective for FIRES patients. More studies are needed to reveal the exact mechanism of $\mathrm{KD}$ in FIRES, define the optimal timing and propose a protocol of $\mathrm{KD}$ application.

\section{CONCLUSIONS}

In summary, this study shows that $\mathrm{KD}$ has a therapeutic effect on FIRES, especially in the acute phase as it can resolve SRSE. Early identification of FIRES and prompt initiation of $\mathrm{KD}$ therapy may improve the prognosis 
of this condition. Unique EEG features of FIRES may aid early diagnosis. Intravenous $\mathrm{KD}$ is a reasonable alternative for patients who cannot take $\mathrm{KD}$ enterally due to intestinal failure.

\section{AUTHOR CONTRIBUTIONS}

PP, LM, and JP conceived the study. XD and FH provided the clinical information and PP checked. CC, XW, and JP analyzed the EEG data. PP drafted the initial manuscript which was edited by LM, SG, FY, and JP.

\section{REFERENCES}

1. Hirsch LJ, Gaspard N, van Baalen A, Nabbout R, Demeret S, Loddenkemper $\mathrm{T}$, et al. Proposed consensus definitions for new-onset refractory status epilepticus (NORSE), febrile infection-related epilepsy syndrome (FIRES), and related conditions. Epilepsia. (2018) 59:739-44. doi: 10.1111/epi. 14016

2. van Baalen A, Vezzani A, Häusler M, Kluger G. Febrile infection-related epilepsy syndrome: clinical review and hypotheses of epileptogenesis. Neuropediatrics. (2017) 48:5-18. doi: 10.1055/s-0036-1597271

3. Hon KL, Leung A, Torres AR. Febrile infection-related epilepsy syndrome (fires): an overview of treatment and recent patents. Recent Pat Inflamm Allergy Drug Discov. (2018) 12:12835. doi: 10.2174/1872213X12666180508122450

4. Klepper J. Glucose transporter deficiency syndrome (GLUT1DS) and the ketogenic diet. Epilepsia. (2008) 49:469. doi: 10.1111/j.1528-1167.2008.01833.x

5. Yang H, Wu J, Guo R, Peng Y, Zheng W, Liu D, et al. Glycolysis in energy metabolism during seizures. Neural Regen Res. (2013) 8:1316-26. doi: 10.3969/j.issn.1673-5374.2013.14.008

6. Liu F, Peng J, Zhu C, Xiao H, He F, Yin F, et al. Efficacy of the ketogenic diet in Chinese children with Dravet syndrome: a focus on neuropsychological development. Epilepsy Behav. (2019) 92:98-102. doi: 10.1016/j.yebeh.2018.12.016

7. Nabbout R, Mazzuca M, Hubert P, Peudennier S, Allaire C, Flurin V, et al. Efficacy of ketogenic diet in severe refractory status epilepticus initiating fever induced refractory epileptic encephalopathy in school age children (FIRES). Epilepsia. (2010) 51:2033-7. doi: 10.1111/j.1528-1167.2010. 02703.x

8. Chang P, Augustin K, Boddum K, Williams S, Sun M, Terschak JA, et al. Seizure control by decanoic acid through direct AMPA receptor inhibition. Brain. (2016) 139:431-43. doi: 10.1093/brain/awv325

9. Dupuis N, Curatolo N, Benoist JF, Auvin S. Ketogenic diet exhibits antiinflammatory properties. Epilepsia. (2015) 56:e95-8. doi: 10.1111/epi.13038

10. Singh RK, Joshi SM, Potter DM, Leber SM, Carlson MD, Shellhaas RA. Cognitive outcomes in febrile infection-related epilepsy syndrome treated with the ketogenic diet. Pediatrics. (2014) 134:e1431-5. doi: 10.1542/peds.2013-3106

11. Nangia S, Caraballo RH, Kang HC, Nordli DR, Scheffer IE. Is the ketogenic diet effective in specific epilepsy syndromes? Epilepsy Res. (2012) 100:2527. doi: 10.1016/j.eplepsyres.2012.01.015

12. Kossoff EH, Zupec-Kania BA, Auvin S, Ballaban-Gil KR, Christina $\mathrm{BA}$, Blackford $\mathrm{R}$, et al. Optimal clinical management of children receiving dietary therapies for epilepsy: Updated recommendations of the International Ketogenic Diet Study Group. Epilepsia Open. (2018) 3:175-92. doi: 10.1002/epi4.12225

13. Kramer U, Chi CS, Lin KL, Specchio N, Sahin M, Olson H, et al. Febrile infection-related epilepsy syndrome (FIRES): pathogenesis, treatment, and

\section{FUNDING}

This study was funded by the program the National Natural Science Foundation of China (NO. 81771409).

\section{ACKNOWLEDGMENTS}

We thank the patients and their families for the participation in our study, and clinicians for referring patients. We also acknowledge the support of Guangzhou Kinton Medical Food. Ltd.

outcome: a multicenter study on 77 children. Epilepsia. (2011) 52:1956-65. doi: 10.1111/j.1528-1167.2011.03250.x

14. Howell KB, Katanyuwong K, Mackay MT, Bailey CA, Scheffer IE, Freeman JL, et al. Long-term follow-up of febrile infection-related epilepsy syndrome. Epilepsia. (2012) 53:101-10. doi: 10.1111/j.1528-1167.2011.03350.x

15. Betjemann JP, Josephson SA, Lowenstein DH, Burke JF. Trends in status epilepticus-related hospitalizations and mortality: redefined in us practice over time. Jama Neurol. (2015) 72:650-5. doi: 10.1001/jamaneurol.2015.0188

16. Payne ET, Zhao XY, Frndova H, McBain K, Sharma R, Hutchison JS, et al. Seizure burden is independently associated with short term outcome in critically ill children. Brain. (2014) 137:1429-38. doi: 10.1093/brain/awu042

17. Caraballo RH, Reyes G, Avaria MFL, Buompadre MC, Gonzalez M, Fortini $\mathrm{S}$, et al. Febrile infection-related epilepsy syndrome: a study of 12 patients. Seizure. (2013) 22:553-9. doi: 10.1016/j.seizure.2013.04.005

18. Sort R, Born AP, Pedersen KN, Fonsmark L, Uldall P. Ketogenic diet in 3 cases of childhood refractory status epilepticus. Eur J Paediatr Neuro. (2013) 17:531-6. doi: 10.1016/j.ejpn.2013.05.001

19. Cervenka MC, Hartman AL, Venkatesan A, Geocadin RG, Kossoff EH. The ketogenic diet for medically and surgically refractory status epilepticus in the neurocritical care unit. Neurocrit Care. (2011) 15:51924. doi: 10.1007/s12028-011-9546-3

20. Farias-Moeller R, Bartolini L, Pasupuleti A, Brittany Cines RD, Kao A, Carpenter JL. A practical approach to ketogenic diet in the pediatric intensive care unit for super-refractory status epilepticus. Neurocrit Care. (2017) 26:267-72. doi: 10.1007/s12028-016-0312-4

21. Rivas-Coppola MS, Shah N, Choudhri AF, Morgan R, Wheless JW. Chronological evolution of magnetic resonance imaging findings in children with febrile infection-related epilepsy syndrome. Pediatr Neurol. (2016) 55:22-9. doi: 10.1016/j.pediatrneurol.2015.09.003

22. Vaccarezza M, Silva W, Maxit C, Agosta G. Super-refractory status epilepticus: treatment with ketogenic diet in pediatrics (Spanish). Rev Neurol. (2012) 55:20-5.

23. Chiusolo F, Diamanti A, Bianchi R, Fusco L, Elia M, Capriati T, et al. From intravenous to enteral ketogenic diet in PICU: a potential treatment strategy for refractory status epilepticus. Eur J Paediatr Neuro. (2016) 20:8437. doi: 10.1016/j.ejpn.2016.08.004

Conflict of Interest Statement: The authors declare that the research was conducted in the absence of any commercial or financial relationships that could be construed as a potential conflict of interest.

Copyright (c) 2019 Peng, Peng, Yin, Deng, Chen, He, Wang, Guang and Mao. This is an open-access article distributed under the terms of the Creative Commons Attribution License (CC BY). The use, distribution or reproduction in other forums is permitted, provided the original author(s) and the copyright owner(s) are credited and that the original publication in this journal is cited, in accordance with accepted academic practice. No use, distribution or reproduction is permitted which does not comply with these terms. 